\title{
Endogenous endophthalmitis: a 9-year retrospective study at a tertiary referral hospital in Malaysia
}

\author{
Rosiah Muda', Valarmathy Vayavari ${ }^{2}$, Deivanai Subbiah ${ }^{3}$, Hamisah Ishak ${ }^{4}$ Azian Adnan $^{3}$ \\ and Shelina Oli Mohamed ${ }^{5 *}$
}

\begin{abstract}
Background: The objective of this study was to determine the clinical presentation, systemic risk factors, source of infective microorganism, treatment outcomes, and prognostic indicators of endogenous endophthalmitis at a main tertiary referral hospital for uveitis in Malaysia. A retrospective review of medical records of 120 patients (143 eyes) with endogenous endophthalmitis over a period of 9 years between January 2007 and December 2015 was undertaken.

Results: Identifiable systemic risk factors were present in 79.2\%, with the majority related to diabetes mellitus ( 60. $0 \%$ ). The most common source of bacteremia was urinary tract infection (17.5\%). A positive culture from ocular fluid or other body fluids was obtained in 82 patients (68.9\%), and the blood was the highest source among all culturepositive results (42.0\%). Gram-negative organisms accounted 42 cases (50.6\%) of which Klebsiella pneumonia was the most common organism isolated (32.5\%). Sixty-nine eyes (48.6\%) were managed medically, and 73 eyes (51.4\%) underwent vitrectomy. Final visual acuity of counting fingers (CF) or better was achieved in 100 eyes (73.0\%). Presenting visual acuity of CF or better was significantly associated with a better final acuity of CF or better ( $p=0.001)$.

Conclusions: The visual prognosis of endogenous endophthalmitis is often poor, leading to blindness. As expected, gram-negative organisms specifically Klebsiella pneumonia were the most common organisms isolated. Urinary tract infection was the main source of infection. Poor presenting visual acuity was significantly associated with grave visual outcomes. A high index of suspicion, early diagnosis, and treatment are crucial to salvage useful vision.
\end{abstract}

Keywords: Endogenous, Endophthalmitis, Diabetes mellitus, Bacteria, Fungal, Intravitreal injections, Vitrectomy, Visual acuity

\section{Background}

Endogenous endophthalmitis (EE) occurs when infectious agents are hematogenously disseminated into the eye from a remote focus of infection. Even though this entity is relatively rare and accounts for approximately $2-15 \%$ of all cases of endophthalmitis [1-3], it is an ocular emergency and is potentially life-threatening. The causative organisms may vary depending on the geographical location. In Europe and the USA, Streptococcus species, Staphylococcus aureus, and other gram-positive bacteria account for two thirds of bacterial endogenous

\footnotetext{
* Correspondence: shelin06@gmail.com

${ }^{5}$ Department of Opthalmology, Hospital Shah Alam, Shah Alam, Malaysia Full list of author information is available at the end of the article
}

endophthalmitis cases and gram-negative isolates are found in only $32 \%$ of cases [3, 4]. In contrast, most cases of EE in East Asia are caused by gram-negative organisms especially Klebsiella species accounting for 80 to $90 \%$ of positive cultures $[4,5]$.

The outcome of endogenous endophthalmitis is often dismal. Sharma et al. reported that $60 \%$ of the eyes had a final visual acuity of hand motions or worse and as many as $29 \%$ required removals [6]. Hence, prompt diagnosis and management are essential if useful vision is to be preserved.

To the best of our knowledge, there is no large case series on endogenous endophthalmitis being reported yet from Malaysia. The current study was performed to 
determine the clinical profile of EE at a tertiary hospital while focusing on the clinical presentation, predisposing risk factors, source of infective microorganism, treatment outcomes and prognostic indicators.

\section{Methods}

A retrospective observational study was conducted in Selayang Hospital which was the main national tertiary referral center for uveitis in Malaysia. We reviewed the medical records of patients with endogenous endophthalmitis who were seen or referred to our hospital over a period of 9 years between January 2007 and December 2015. The diagnosis of endogenous endophthalmitis was defined as the presence of iritis and vitritis on ophthalmic examination and one or more of the following: (1) constitutional symptoms and systemic infection; (2) positive cultures of vitreous, blood, or other body fluids; (3) presence of loculation, vitreous debris, or membranous debris on ultrasound; (4) lack of ocular trauma or ocular surgery within 1 year from onset of infection or evidence of primary external ocular infection such as infectious keratitis or filtering bleb infection.

Demographic details such as age, gender and race, presenting complaints, preexisting medical illnesses, predisposing risk factors, source of infection, laterality, visual acuity, ophthalmologic examination, ultrasound findings, microbiologic profiles, treatment modalities, and final visual outcomes were collected from medical records.

The study was done according to Malaysian Good Clinical Practice (MGCP) 2nd edition January 2004 and registered in National Medical Research Register (NMRR).

Data was analyzed using the Statistical Package for Social Science (SPSS) version 22.0. Descriptive data was expressed as mean \pm standard deviation (SD) for numerical data, and categorical variables were presented in frequencies and percentages. Logistic regression analysis was used to determine the factors associated with good visual outcomes. The association between presenting and final visual acuity was also analyzed using the Pearson correlation coefficient, and visual acuities were converted to logarithm of the minimal angle of resolution ( $\log$ MAR) scale. For visual acuity less than counting finger $(\mathrm{CF})$, the following scales were used: $\mathrm{CF}=2.00 \mathrm{Log}$ MAR units, hand motion $=2.30$ LogMAR units, light perception $=2.60$ LogMAR units, and no light perception $=2.90$ LogMAR units. A $P$ value of $<0.05$ was considered to be significant.

\section{Results}

\section{Demographic data}

A total of 143 eyes of 120 patients were included in this study. The age of patients ranged from 7 to 81 years, and the mean age at presentation was $52.6 \pm 15.1$ years. The racial distribution reflected the multiracial population in our country with 72 Malays (60.0\%), 33 Chinese (27.5\%), 13 Indian (10.8\%), and 2 others (1.7\%). There was a slight female predominance $(61,50.8 \%)$ compared to males $(59,49.2 \%)$.

\section{Systemic features \\ Systemic risk factors}

At least one underlying medical illness was identified in 95 patients (79.2\%). Diabetes mellitus was the most common medical illness (72, 60.0\%), followed by renal failure (20, 16.7\%), and 15 patients (12.5\%) had solid organ tumor or hematologic malignancy. Six patients (5.0\%) had liver disease and 3 patients (2.5\%) were pregnant. Five patients $(4.2 \%)$ were on systemic corticosteroids for underlying autoimmune diseases, and 1 was on systemic immunosuppressants.

\section{Source of infection}

A primary source of infection was identified in 90 patients (75.0\%). Urinary tract infection $(21,17.5 \%)$ was the most common source of bacteremia followed by pulmonary infection $(19,15.8 \%)$, skin or soft tissue infection (17, $14.2 \%)$, and hepatobiliary infection (12, 10.0\%). However, the source of infection could not be identified in 30 patients (25.0\%), despite extensive systemic work-up and investigations (Table 1).

\section{Ocular features}

Ocular symptoms Majority of patients had unilateral disease, 97 (80.8\%), and involvement of the left eye (78, $54.5 \%$ ) was more common. Blurring of vision (106, $74.1 \%)$, eye redness $(52,36.4 \%)$, eye pain or discomfort (42, $29.4 \%)$, and floaters $(16,11.2 \%)$ were ocular symptoms at presentation. The blurring of vision $(85,68.5 \%)$ was the most common presenting complaint, followed

Table 1 Identifiable source of infection

\begin{tabular}{lll}
\hline Source of infection & No. of patients & Percent \\
\hline Infected catheter & 11 & 9.2 \\
Urinary tract infection & 21 & 17.5 \\
Hepatobiliary infection & 12 & 10.0 \\
Lung infection & 19 & 15.8 \\
Meningitis & 2 & 1.7 \\
Infective endocarditis & 1 & 0.8 \\
Gastrointestinal infection & 1 & 0.8 \\
Genital infection & 2 & 1.7 \\
Skin or soft tissue infection & 17 & 14.2 \\
Septic arthritis & 1 & 0.8 \\
Diabetic foot ulcer & 3 & 2.5 \\
Nil & 30 & 25.0 \\
\hline
\end{tabular}


by eye redness $(20,16.1 \%)$ and eye pain or discomfort $(14,11.3 \%)$.

The interval between the onset of ocular symptoms and the first presentation to an ophthalmologist was less than 1 week in 62 eyes (47.3\%), 1 to 2 weeks in 40 eyes (30.5\%), more than 2 weeks to 1 month in 10 eyes (14.5\%), and more than 1 month in 10 eyes (7.6\%). The interval was not available in 12 eyes which were from patients with no recorded ocular symptoms due to poor general medical condition or no documentation obtained from the medical records (Fig. 1).

Systemic symptoms were identified in 84 patients (70.0\%). This data was not available in 36 patients (30.0\%). The interval between the onset of systemic symptoms and the onset of ocular symptoms was identified in 101 eyes. The interval was less than 1 week in 26 eyes (25.7\%), between 1 and 2 weeks in 25 eyes (24.8\%), more than 2 weeks to 1 month in 12 eyes (11.9\%), and more than 1 month in 38 eyes (37.6\%) (Fig. 1).

Ocular findings At presentation, based on the Standardization of Uveitis Nomenclature (SUN), the proportion of the eyes with anterior chamber cells better than grade 3 and grade 3 or worse was similar with 67 eyes (49.6\%) and 68 eyes (50.4\%) respectively. Twentyseven eyes (19.0\%) had hypopyon, and 42 eyes (29.6\%) had fibrin. The fundal view was present in 64 out of 143 eyes $(44.8 \%)$. Among this, 32 eyes $(50.0 \%)$ had choroiditis or choroidal abscess, 11 eyes $(17.2 \%)$ had retinitis, 6 (9.4\%) had vasculitis, and 4 (6.3\%) had optic disc swelling. Six eyes had a combination of choroiditis or choroidal abscess and retinitis and 2 eyes had choroiditis or choroidal abscess, vasculitis, and optic disc swelling.

Ultrasound findings were documented in 97 eyes. Fifty-five eyes (56.7\%) had vitreous loculation, whereas subretinal or vitreous abscess and retinal detachment were found in $6(6.2 \%)$ and $8(8.2 \%)$ eyes respectively.

\section{Microbiology}

A positive culture from ocular fluid or other body fluids was obtained in 82 patients (68.9\%). The culture result of 1 patient was not available as he had it done elsewhere.

The blood was the highest source among all culturepositive results (50, 42.0\%). Gram-negative organisms were more common (26 patients, 52.0\%) than gram-positive organisms (20 patients, $40.0 \%)$. Four patients $(8.0 \%)$ had a positive fungal culture from the blood (Fig. 2).

Forty-six patients (38.7\%) had at least one positive culture from other body fluids. Other body fluid cultures yielded gram-positive organisms in 11 patients (23.9\%), gramnegative in 28 patients $(23.3 \%)$, and fungal in 7 patients $(15.2 \%)$. Nineteen patients (41.3\%) had a positive urine culture; $14(30.4 \%)$ had positive cultures from infected catheter, skin, soft tissue, or joint; 8 (17.4\%) had positive sputum cultures; 5 (10.9\%) had positive culture from liver abscess; 3 (6.5\%) had positive high vaginal swab; and 1 patient (2.1\%) had positive cerebrospinal fluid (Figs. 2 and 3).

Vitreous samples were obtained from 125 eyes. Vitreous culture was positive in 27 eyes (22.3\%). Of these, 22 eyes (81.4\%) had bacterial and 5 eyes (18.5\%) had fungal isolates. Among bacterial isolates, $10(37.0 \%)$ were grampositive and 12 (44.4\%) were gram-negative (Fig. 2).

The causative organisms cultured from the blood, vitreous, and other body fluids are summarized in Table 2.

The microbiology of causative organisms is summarized in Table 3.

\section{Treatment}

All patients were treated with systemic antibiotics or antifungal agents aimed at the source of infection and presumed causative organism. One hundred nineteen cases (85.6\%) were on systemic antibiotics, and more than half (55.1\%) were treated with ciprofloxacin either as monotherapy or in combination with other antibiotics. There was no statistically significant correlation

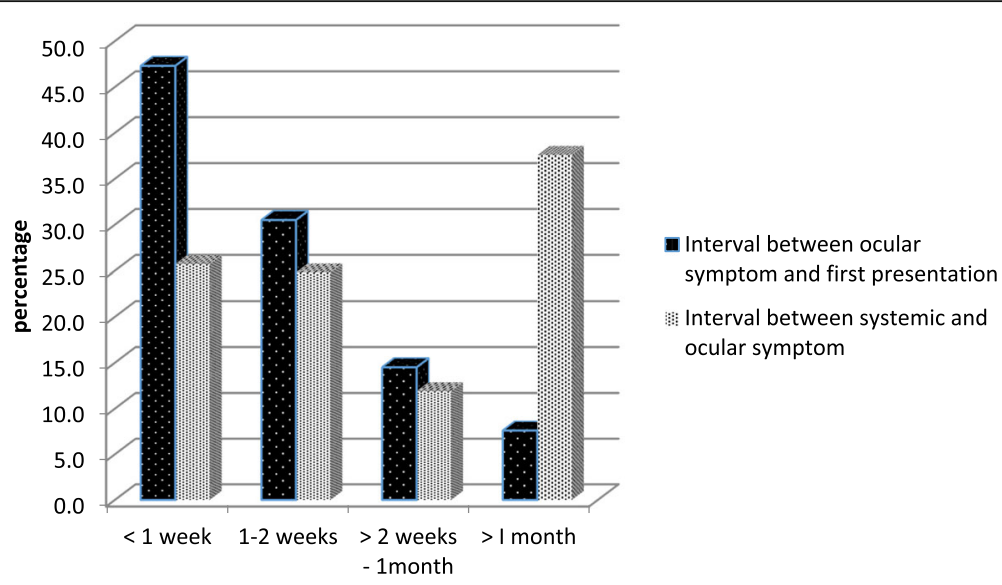

Fig. 1 The interval between the onset of ocular symptom and first presentation and the duration between systemic and ocular symptom 


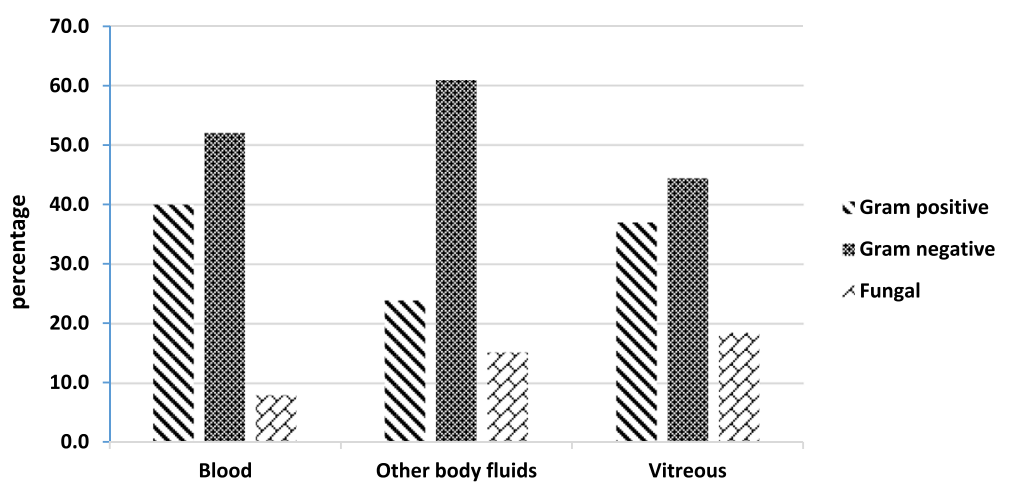

Fig. 2 Blood, other body fluids, and vitreous culture organism

between systemic ciprofloxacin and final visual outcomes $(p=0.68)$. Systemic steroids were not used in any of the patients. In addition, 126 eyes (88.7\%) received intravitreal antibiotics (vancomycin and ceftazidime or amikacin) or antifungal (amphotericin B) injections or both. The injections were repeated in 75 eyes (59.5\%). Intravitreal injection was not given in 16 cases (11.3\%), and 10 of them $(62.5 \%)$ had relatively good presenting visual acuity of $6 / 24$ or better and mild vitritis. They were treated with systemic antibiotics or antifungals with close monitoring. No intravitreal injection of steroids was given to any eye. Overall, 69 eyes (48.6\%) were managed medically either with systemic or intravitreal antibiotics or antifungals or both.

Out of 143 eyes, 73 (51.4\%) underwent vitrectomy. Early vitrectomy was performed within 2 weeks from diagnosis in 38 eyes (52.1\%). Silicone oil was injected in 35 eyes (47.9\%), gas in 3 eyes (4.1\%), air in 19 eyes (26.0\%), and no intraocular tamponade was used in 16 eyes $(21.9 \%)$.

\section{Outcomes}

The most common presenting visual acuity was between 6/60 and counting finger (CF) (48 eyes, 34.0\%), followed by hand motion $(38,27.0 \%)$ and perception of light (22 eyes, $15.6 \%)$. Only 16 eyes (11.3\%) and 14 eyes (9.9\%) had presented visual acuity between $6 / 6$ to $6 / 18$ and $6 /$ 24 to $6 / 36$ respectively. Overall, $56 \%$ had vision of CF or better at presentation. Vision was not available in 2 patients (1.4\%) who could not cooperate during a vision test. Final visual acuity was available in 138 eyes (96.5\%). Three patients were transferred to their original hospitals for the continuation of treatment and follow-up. One patient defaulted follow-up, and another patient passed away due to sepsis and multiorgan failure.

After treatment, 100 eyes (73.0\%) achieved final visual acuity of CF or better. Ten eyes developed panophthalmitis, and 6 eyes required evisceration. Table 4 summarizes the logistic regression analysis results of patient characteristics that may predict a good visual outcome (CF or better).

In univariate logistic regression analysis, factors found to be statistically significant with good visual outcome were good presenting visual acuity (crude odd ratio 0.1; 95\% CI $0.021,0.384$ ) and presence of fundus view at presentation (crude odd ratio 0.337; 95\% CI 0.068,1.675). In the multivariate logistic regression analysis, elevated risk for good visual outcome was observed only in good

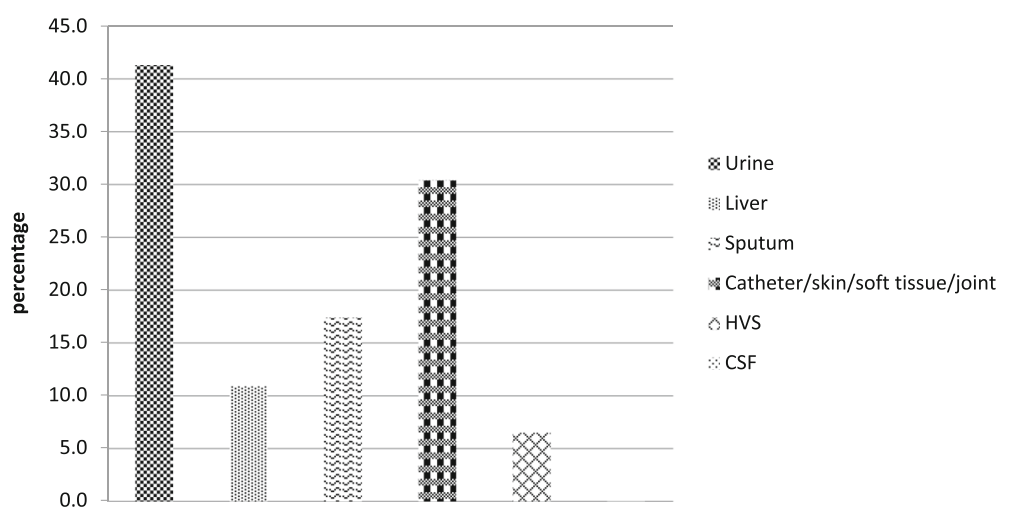

Fig. 3 Other body fluid culture 
Table 2 Microbial isolates from blood, other body fluids, and vitreous

\begin{tabular}{|c|c|c|c|}
\hline Species & Blood ( $n=$ patient $)$ & Other body fluids ( $n=$ patient) & Vitreous ( $n=$ eyes) \\
\hline Culture positive & $50(42.0 \%)$ & $46(38.7 \%)$ & $27(22.3 \%)$ \\
\hline Culture negative & 69 (58.0\%) & $73(61.3 \%)$ & $94(77.7 \%)$ \\
\hline Gram-positive organism & $20(40.0 \%)$ & $11(23.9 \%)$ & $10(37.0 \%)$ \\
\hline Staphylococcus aureas & $13(26.0 \%)$ & $7(15.2 \%)$ & $5(18.5 \%)$ \\
\hline MRSA & $3(6.0 \%)$ & $2(4.3 \%)$ & $2(7.4 \%)$ \\
\hline Staphylococcus coagulase -ve & $2(4.0 \%)$ & $0(0.0 \%)$ & $2(7.4 \%)$ \\
\hline Streptococcus sp. & $2(4.0 \%)$ & $2(4.3 \%)$ & $1(3.7 \%)$ \\
\hline Gram-negative organism & $26(52.0 \%)$ & $28(60.9 \%)$ & $12(44.4 \%)$ \\
\hline Klebsiella pneumonia & 17 (34.0\%) & $19(41.3 \%)$ & $8(29.6 \%)$ \\
\hline Pseudomonas aeruginosa & $2(4.0 \%)$ & $4(8.7 \%)$ & $3(11.1 \%)$ \\
\hline Escherichia coli & $1(2.0 \%)$ & $3(6.5 \%)$ & $0(0.0 \%)$ \\
\hline Acinebacter sp. & $1(2.0 \%)$ & $2(4.3 \%)$ & $0(0.0 \%)$ \\
\hline Enterobacter intermedius & $1(2.0 \%)$ & $0(0.0 \%)$ & $0(0.0 \%)$ \\
\hline Bukholderia cepacia & $1(2.0 \%)$ & $0(0.0 \%)$ & $1(3.7 \%)$ \\
\hline Bukholderia pseudomallei & $1(2.0 \%)$ & $0(0.0 \%)$ & $0(0.0 \%)$ \\
\hline Elizabethkingia meningosepticum & $1(2.0 \%)$ & $0(0.0 \%)$ & $0(0.0 \%)$ \\
\hline Mycoplasma pneumonia & $1(2.0 \%)$ & $0(0.0 \%)$ & $0(0.0 \%)$ \\
\hline Fungal & $4(8.0 \%)$ & $7(15.2 \%)$ & $5(18.5 \%)$ \\
\hline Candida albicans & $1(2.0 \%)$ & $5(10.9 \%)$ & $1(3.7 \%)$ \\
\hline Candida tropicalis & $2(4.0 \%)$ & $1(2.2 \%)$ & $0(0.0 \%)$ \\
\hline Penicillium sp. & $0(0.0 \%)$ & $0(0.0 \%)$ & $2(7.4 \%)$ \\
\hline Phanerochaeta chrysosporium & $0(0.0 \%)$ & $0(0.0 \%)$ & $1(3.7 \%)$ \\
\hline Xylariaceae sp. & $0(0.0 \%)$ & $0(0.0 \%)$ & $1(3.7 \%)$ \\
\hline Fungal (species not available) & $1(2.0 \%)$ & $1(2.2 \%)$ & $0(0.0 \%)$ \\
\hline
\end{tabular}

presenting visual acuity (adjusted odd ratio 0.09; 95\% CI $0.021,0.384)$. We also found a moderate correlation between presenting visual acuity and final visual acuity (Pearson $r=0.564, p<0.001$ (Fig. 4).

Gender, age group, presence of underlying medical illness, existing DM, source of infection, culture positivity, types of organism, intravitreal antibiotics, vitrectomy, or early vitrectomy were not significantly associated with good final visual outcomes.

\section{Discussion}

In this study, we wanted to determine whether the clinical profiles of EE at a tertiary hospital in Malaysia were similar to those reported from other countries.

Previous studies had reported a male preponderance with unilateral involvement [7-10]. In contrast, our results showed no difference between male and females.

Predisposing conditions are important in determining a patient's risk for endogenous endophthalmitis. Okada et al. reported $90 \%$ of patients had a positive history of underlying medical conditions such as diabetes, cardiac disease, and malignancy [2]. A major review of endogenous endophthalmitis demonstrated underlying medical conditions predisposing to ocular infection in 56 to $68 \%$ of cases [4]. Another study conducted by Wu and colleagues revealed the identification of preexisting predisposing condition in $90.9 \%$ of patients, and the most common systemic condition found was diabetes mellitus (50\%) [11]. In contrast, Connell et al. reported that intravenous drug abuse was the most common risk factor [1]. Several East Asian studies reported that diabetes mellitus was the most common, and hepatobiliary disease was the second most frequent underlying disease [5, 1214]. In a review of 57 cases of endogenous endophthalmitis in Korea, diabetes mellitus (46.5\%) was the most common underlying disease followed by liver cirrhosis (20.9\%) [15]. In a recent study in which all patients had one or more preexisting medical conditions, the most common was also diabetes mellitus (61.9\%) [16]. Our series revealed similar results, in which diabetes mellitus was the most common systemic disease $(60.0 \%)$ followed by renal failure and malignancy. However, liver diseases were identified only in 6 patients.

In a review of cases by Wong et al., it was reported that hepatobiliary tract infection was the most common source of bacteremia (13 patients, 48\%) [5]. Similar results were 
Table 3 Microbiology of causative organism in endogenous endophthalmitis

\begin{tabular}{lll}
\hline & Number & Percent \\
\hline Gram-positive bacteria & 27 & 32.5 \\
Staphylococcus aureas & 17 & 20.5 \\
MRSA & 3 & 3.6 \\
Staphylococcus coagulase -ve & 4 & 4.8 \\
Streptococcus sp. & 3 & 3.6 \\
Gram-negative bacteria & 42 & 50.6 \\
Klebsiella pneumonia & 27 & 32.5 \\
Pseudomonas aeruginosa & 5 & 6.0 \\
Escherichia coli & 3 & 3.6 \\
Acinebacter sp. & 2 & 2.4 \\
Enterobacter intermedius & 1 & 1.2 \\
Bukholderia cepacia & 1 & 1.2 \\
Bukholderia pseudomallei & 1 & 1.2 \\
Elizabethkingia meningosepticum & 1 & 1.2 \\
Mycoplasma pneumonia & 1 & 1.2 \\
Fungal & 14 & 16.9 \\
Candida albicans & 5 & 6.0 \\
Candida tropicalis & 3 & 3.6 \\
Penicillium sp. & 2 & 2.4 \\
Phanerochaeta chrysosporium & 1 & 1.2 \\
Xylariaceae sp. & 1 & 2.4 \\
Fungal (species not available) & & \\
\hline
\end{tabular}

found in other Korean case series [12, 15, 17]. Interestingly, our case series did not show similar findings with other East Asian reports. We found that urinary tract infection $(21,17.5 \%)$ was the most common source of bacteremia followed by pulmonary infection $(19,15.8 \%)$. Hepatobiliary tract infection was only identified in 12 patients (10.0\%). We also found that among patients who were younger than 40 years old, and older than 60 years old, the most common systemic infection was urinary tract infection at $17.4 \%$ and $30.0 \%$ respectively. In contrast, lung infection (17.5\%) followed by hepatobiliary infection (14.0\%) was the commonest infections among those aged from 40 to 60 years old.

In a case series by Lim et al., the most common presenting complaint was decreased vision $(68.8 \%)$ followed by ocular discomfort (44\%), red eye (20.8\%), and ocular pain (17.4\%) [15]. Ratra et al. also reported that reduced vision (60, 98.4\%), redness $(47,77 \%)$, and pain $(42,68.8 \%)$ were the three most common presenting symptoms [8]. Similar to these studies, our study too revealed blurring of vision, eye redness, and eye pain or discomfort as the main presenting ocular symptoms. However in a case series by Nishida et al., floaters was the second most common ocular symptom after blurring of vision [16].
Ratra et al. in their case series demonstrated that all eyes had severe diffuse endophthalmitis involving the posterior pole. Diffuse vitreous exudates were seen in 47 eyes (77\%). Retina could be visualized in 13 eyes (21.3\%), and $3(4.9 \%)$ had retinal detachment. None had panophthalmitis [8]. In another case series in 18-year review of culture-positive cases in 34 affected eyes, the most common findings were decreased visual acuity (91.1\%), vitritis (79.4\%), conjunctival injection (67.6\%), iritis or retinitis (61.7\%), hypopyon (35.2\%), and retinal detachment (5.8\%) [18]. In our study, 27 eyes (19\%) had hypopyon, 64 eyes (44.8\%) had fundus view, and 8 eyes $(8.2 \%)$ were noted to have retinal detachment on ultrasound. Lower percentage of hypopyon in our patients could be due to the application of topical steroids and antibiotics by the referring ophthalmologist.

The diagnosis of endogenous endophthamitis is typically made following microbiologic evidence of infection from intraocular samples (aqueous or vitreous). Positive cultures from the blood, cerebrospinal fluid, or any extraocular site can be highly suggestive. In our series, the organism causing endophthalmitis was identified by a positive culture from at least one body fluid source in 82 patients $(68.9 \%)$. Blood culture positivity rate varies widely, from 33 to $94 \%$ [4, 19]. Previous large case series have shown higher rates of positivity following blood cultures as compared to vitreous aspirates possibly due to a larger volume sampled $[2,4,11]$. In contrast, Ratra et al. had reported that ocular fluid samples tended to give positive culture results more than blood $(58.6 \%$ vs 3.4\%). This is because all the patients with suspected endogenous endophthalmitis immediately underwent an aqueous tap in the outpatient department before any intravitreal therapy [8]. High rate of positive cultures from intraocular specimens was also demonstrated by Okada et al. (86\%), Binder et al. (70\%), and Ness et al. (81\%) [2, $20,21]$. Vitrectomy has a higher diagnostic yield for culture (92\%) compared to a vitreous aspirate (44\%) [22]. Vitreous samples during vitrectomy were taken near the retinal surface, which can potentially explain the lower yield of needle biopsy. This is because early or localized infection located near the retinal surface might be missed by a needle biopsy [23]. We noted low vitreous yield of organisms in our study. This could be because some of our patients with systemic infection were initially managed by physicians depending on the source of infection. During the time of referral, most of them were already on systemic antibiotics or partially treated. Furthermore, the diagnosis may have been delayed in some while others were generally not stable for early vitreous tapping. Thirty-six patients $(30.0 \%)$ in our series were culture negative.

Causative organisms vary geographically. Studies from the western population revealed that fungal infection was 
Table 4 Prognostic factors associated with good visual outcomes

\begin{tabular}{|c|c|c|c|c|}
\hline Prognostic factor (referent) & Crude odds ratio $(95 \% \mathrm{Cl})$ & $p$ value $^{a}$ & Adjusted odds ratio $(95 \% \mathrm{Cl})$ & $p$ value $^{b}$ \\
\hline \multicolumn{5}{|l|}{ Gender (male) } \\
\hline Female & $2(0.92,4.37)$ & 0.079 & & \\
\hline \multicolumn{5}{|l|}{ Age ( $0-50$ years) } \\
\hline$>50$ years & $0.85(0.39,1.86)$ & 0.679 & & \\
\hline \multicolumn{5}{|l|}{ Medical illness (yes) } \\
\hline No & $1.60(0.55,4.63)$ & 0.383 & & \\
\hline \multicolumn{5}{|l|}{ DM (yes) } \\
\hline No & $2.12(0.93,4.85)$ & 0.071 & & \\
\hline \multicolumn{5}{|l|}{ Presenting VA ( $\geq$ CF) } \\
\hline$<\mathrm{CF}$ & $0.10(0.04,0.25)$ & 0.000 & $0.09(0.021,0.384)$ & 0.001 \\
\hline \multicolumn{5}{|l|}{ Fundus view (yes) } \\
\hline No & $0.15(0.06,0.38)$ & 0.000 & $0.337(0.068,1.675)$ & 0.184 \\
\hline \multicolumn{5}{|l|}{ Source of infection (yes) } \\
\hline No & $2.61(0.93,7.37)$ & 0.062 & & \\
\hline \multicolumn{5}{|l|}{ Culture (positive) } \\
\hline Negative & $1.29(0.54,3.07)$ & 0.566 & & \\
\hline \multicolumn{5}{|l|}{ Organism (gram+) } \\
\hline Gram- & $0.965(0.275-3.386)$ & 0.956 & & \\
\hline Fungal & $0.185(0.019,1.848)$ & 0.151 & & \\
\hline \multicolumn{5}{|l|}{ Intravitreal antibiotic (yes) } \\
\hline No & $2.85(0.62,13.2)$ & 0.234 & & \\
\hline \multicolumn{5}{|l|}{ Vitrectomy (yes) } \\
\hline No & $1.13(0.53-2.41)$ & 0.751 & & \\
\hline \multicolumn{5}{|l|}{ Early vitrectomy ( $\leq 2$ weeks) } \\
\hline$>2$ weeks & $1.69(0.59-4.82)$ & 0.327 & & \\
\hline
\end{tabular}

The clinical summary of patients were summarized in Table 5

a Univariate logistic regression

${ }^{b}$ Only factors associated with good visual outcome in multivariate logistic regression model

the main source in predisposed states, such as intravenous drug abusers and immunocompromised patients [1, 19, 21]. In contrast, gram-negative microbes as the causative organisms were overwhelming in the East Asian experience. In these Asian populations, Klebsiella was found to be responsible for approximately $90 \%$ of all endogenous bacteria endophthalmitis cases [5]. Studies that were conducted in Korea showed that liver abscess was the most common infection source and Klebsiella was the most common causative agent $[15,17]$. A study from Japan in 2015, however, demonstrated that gram-positive organisms were more common $(76.2 \%)$ than gram-negative (19.0\%), contrasted to the findings from other East Asian studies [16]. K. pneumonia which is predominant in East Asia may be due to the high incidence of cholangiohepatitis. Therefore, the East Asian population is more prone to have liver abscess than Caucasians [24]. We found that gram-negative organisms were responsible for half of the cases of endogenous endophthalmitis in our case series
(42 patients, 50.6\%) in which $K$. pneumonia was the most common organism isolated (27 patients, $32.5 \%$ ). Interestingly, in contrast to several East Asian studies, urinary tract infection including renal abscess (9 patients, 33.3\%) was the most common source of infection caused by $K$. pneumonia followed by lung infection (8 patients, 29.6\%) in our series. Liver abscess was identified in 7 patients (25.9\%). Necrotizing fasciitis, infected wound breakdown, and acute gastroenteritis (AGE) were noted in one patient each. Apart from that, there was a relatively higher frequency of gram-positive cocci and fungal infection in our study, $32.5 \%$ and $16.9 \%$ respectively.

Most systemically administered antimicrobials that have been used in the therapy of endophthalmitis do not penetrate well into the non-inflamed vitreous humor. However, the penetration of several antibiotics into the eye may be increased by inflammation which occurs following surgery, trauma, or infection. Kowalski and colleagues compared the minimum inhibitory concentration (MIC) of 


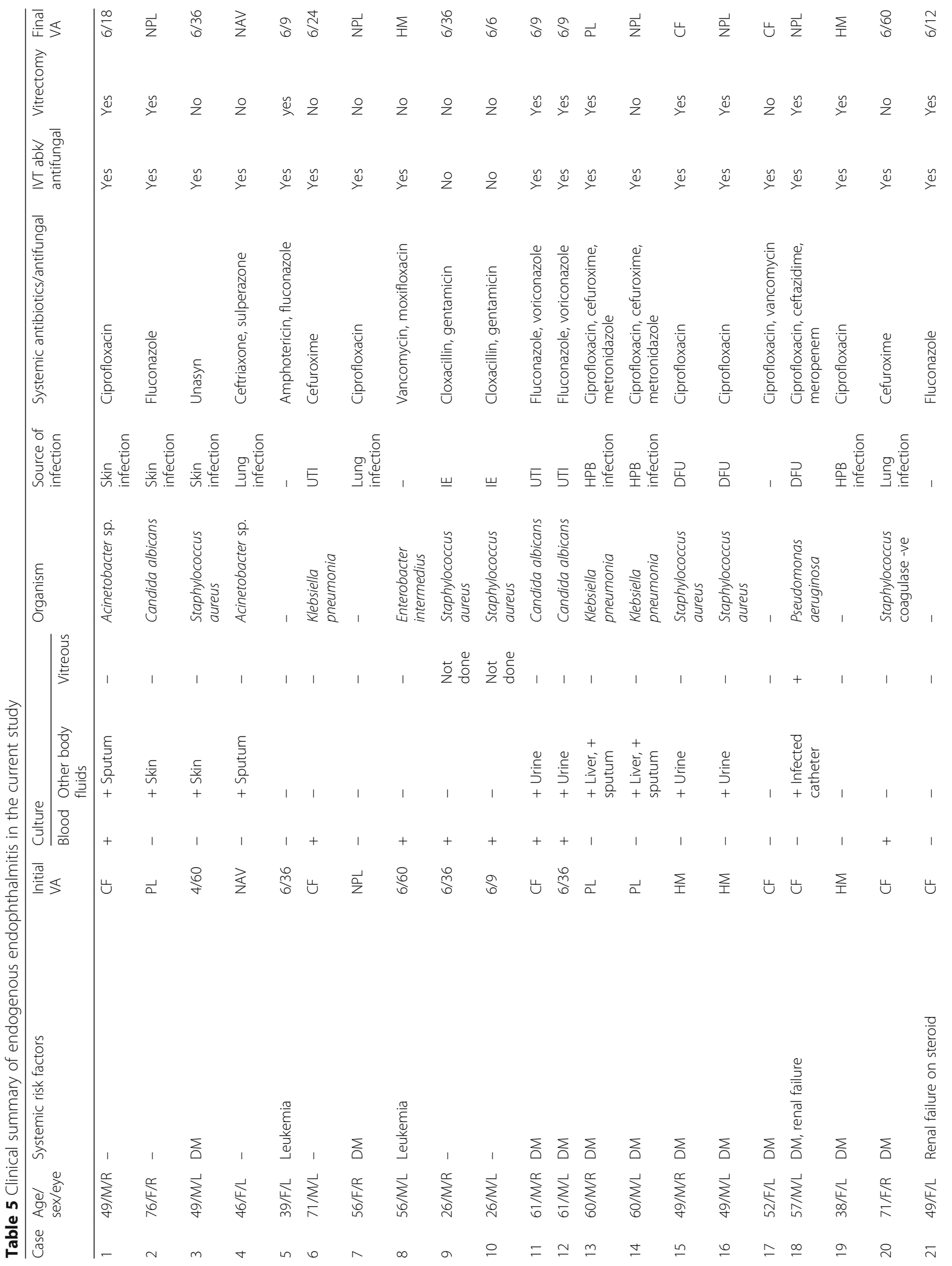




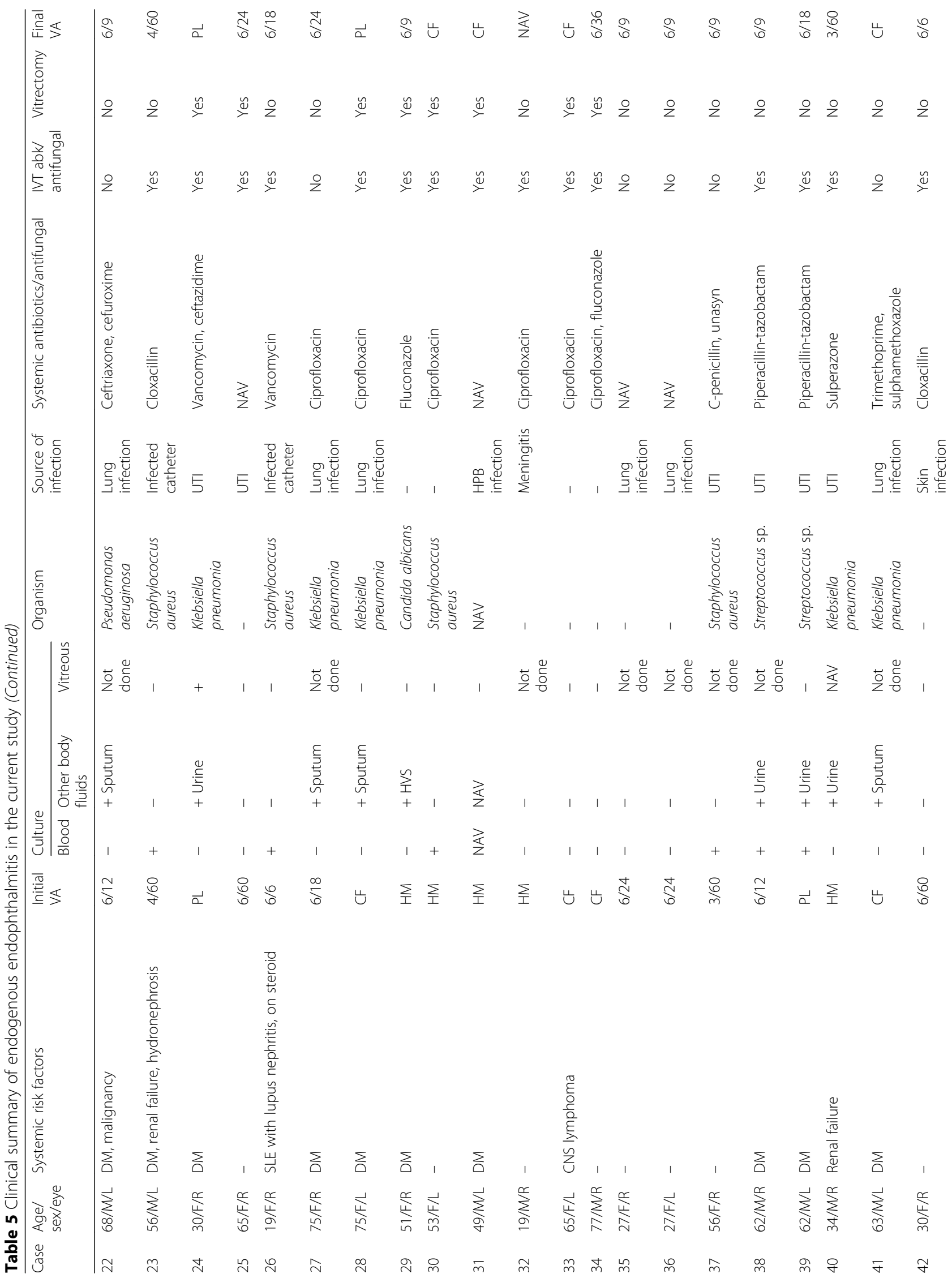




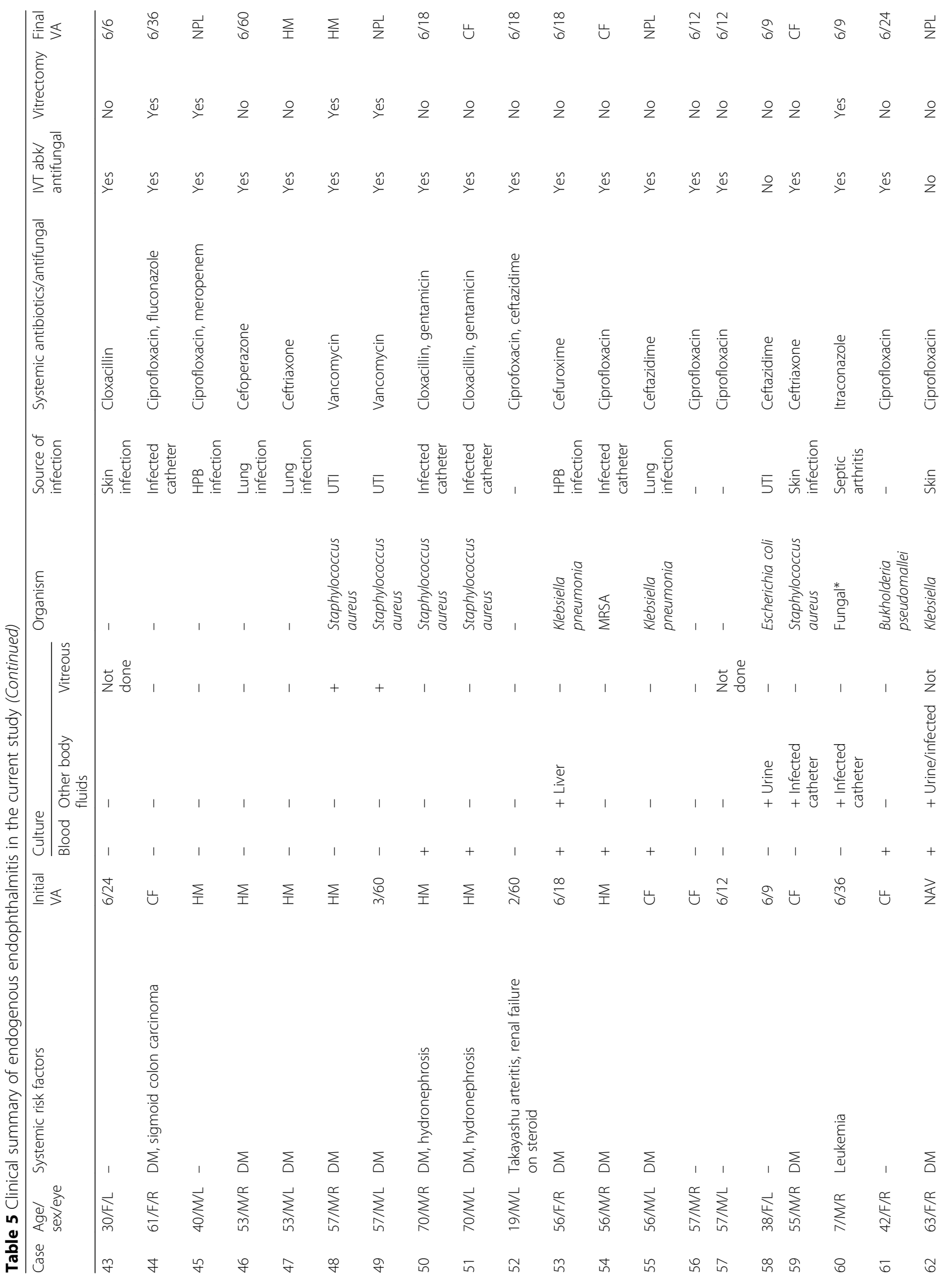




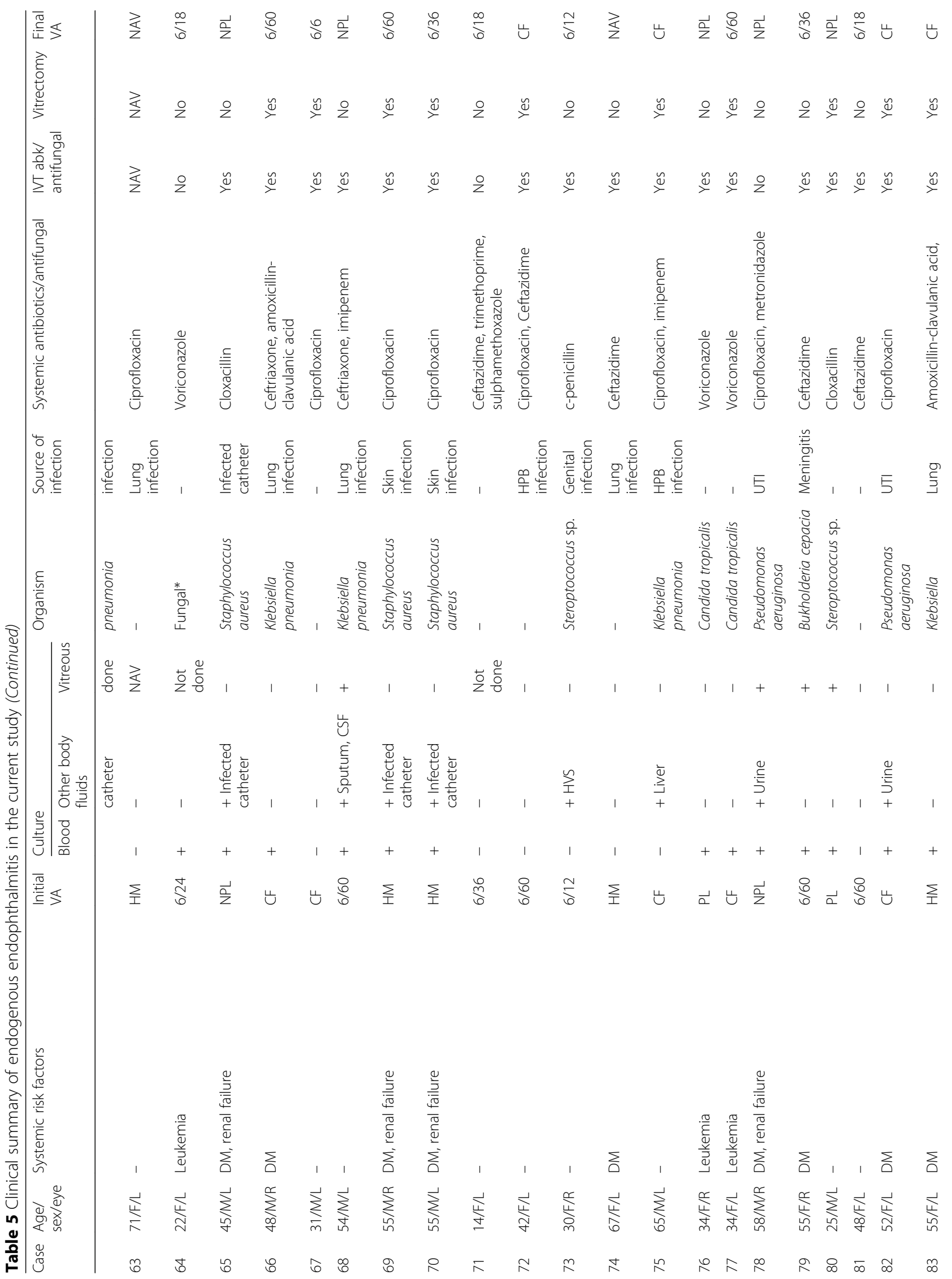




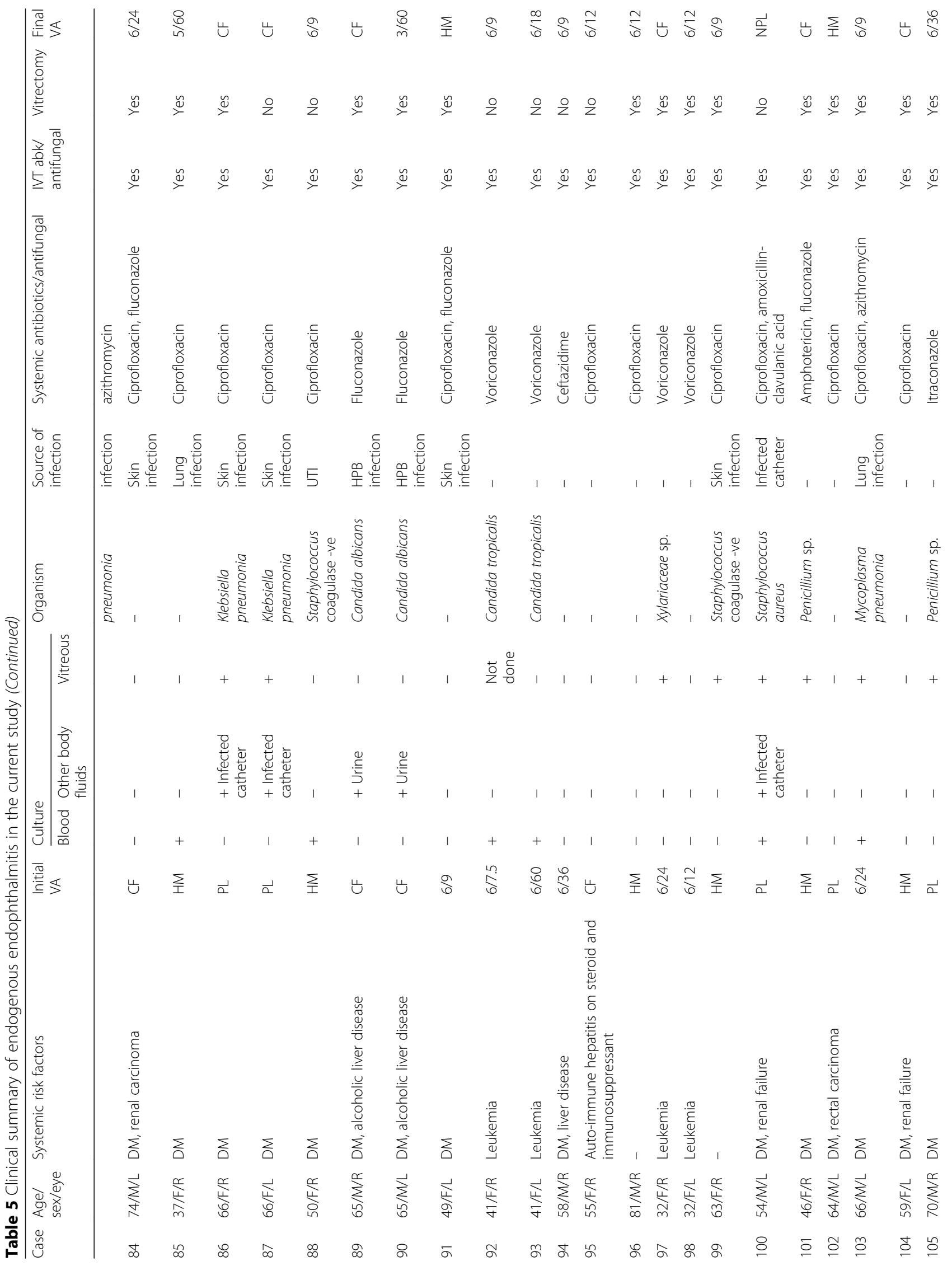




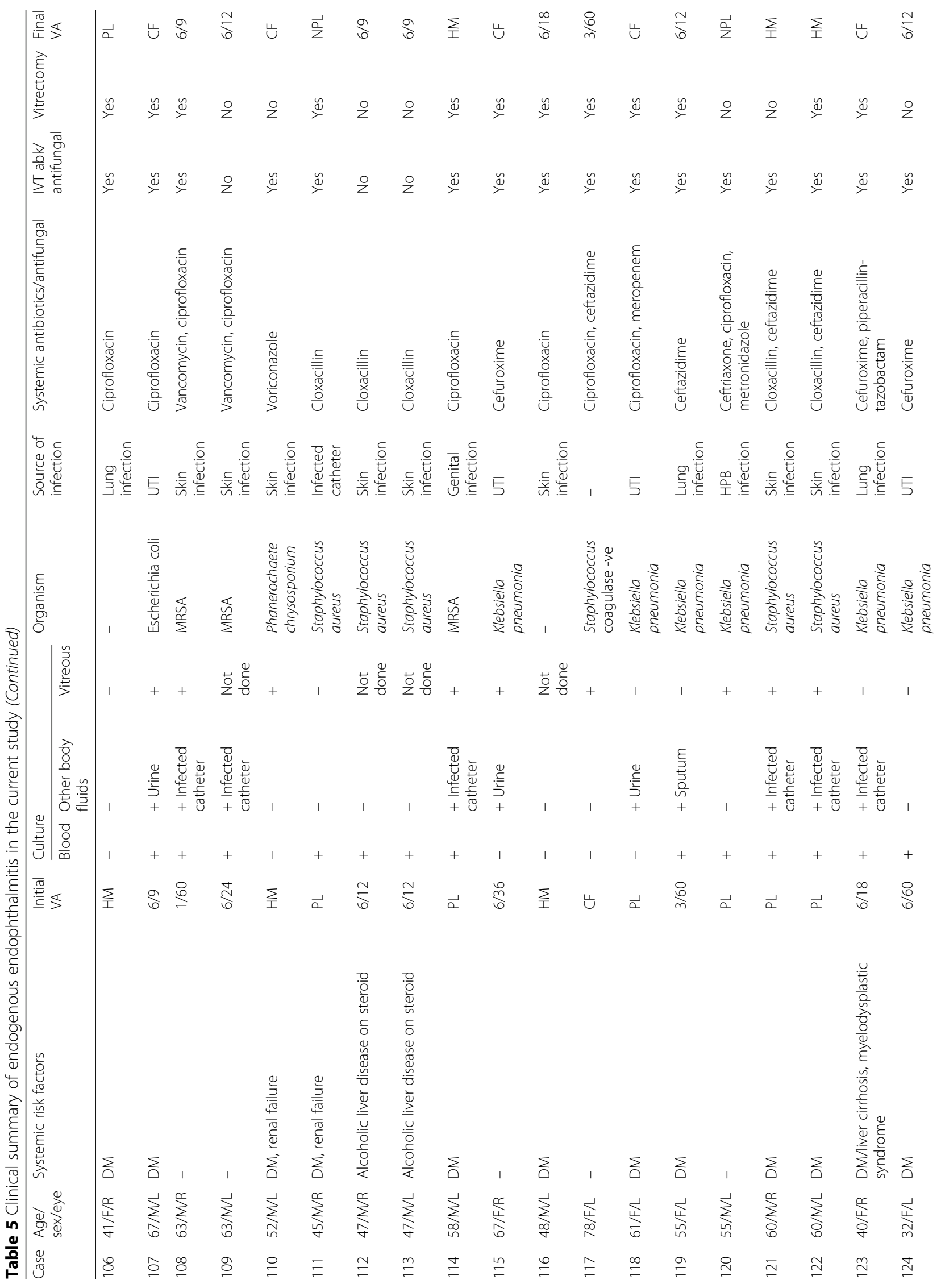


Muda et al. Journal of Ophthalmic Inflammation and Infection (2018) 8:14

Page 14 of 17

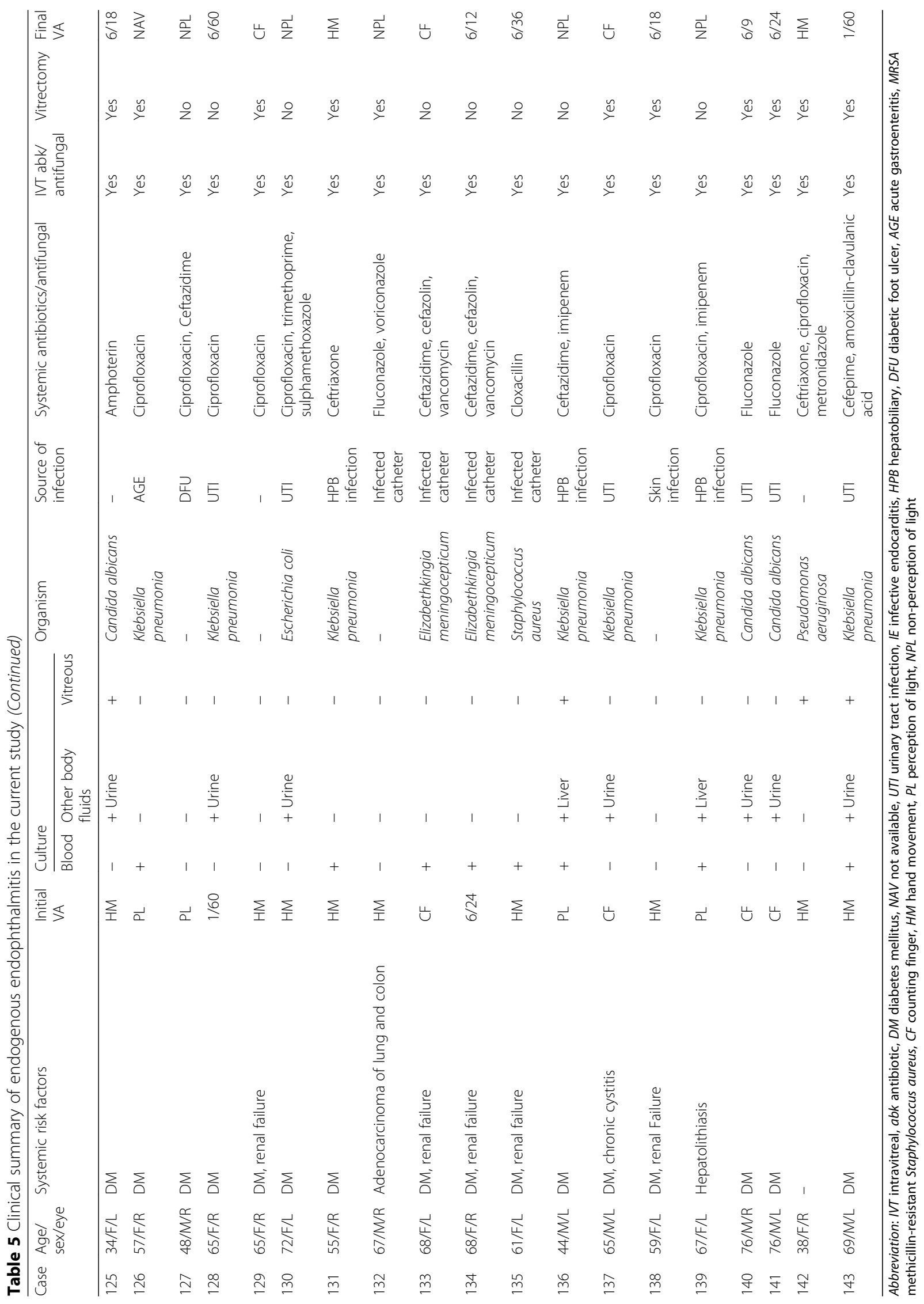




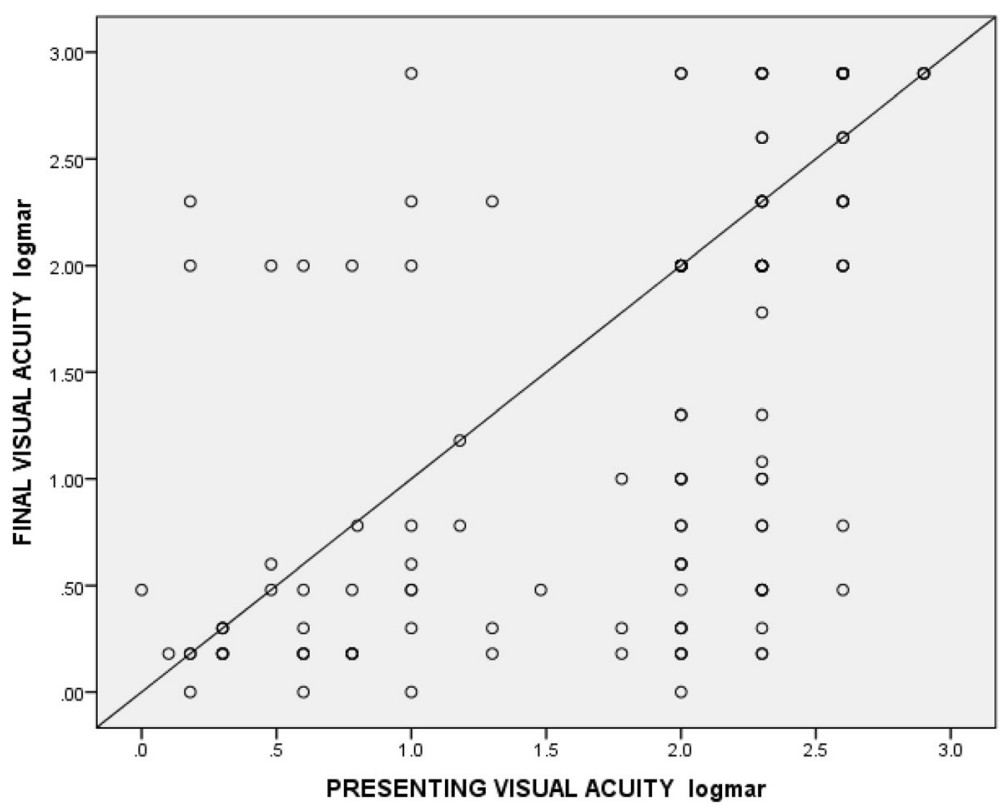

Fig. 4 Correlation between presenting visual acuity (LogMAR) and final visual acuity (LogMAR)

bacterial isolates from 66 patients with endophthalmitis and found that all of the gram-negative isolates would have been inhibited by levels of ciprofloxacin achievable following systemic administration [25].

In endogenous endophthalmitis, the rationale for use of intravitreal injections as an adjunct to intravenous therapy is also because of reduced permeability of the retinal-pigmented epithelium to systemically administered drugs [26]. Yonekawa et al. showed that early administration, e.g., within $24 \mathrm{~h}$, was associated with a favorable visual outcome [27]. Most of our patients received intravitreal injections within $24 \mathrm{~h}$ of diagnosis.

Vitrectomy serves as a diagnostic and therapeutic option. It is indicated in cases with severe vitreous opacities, diffuse retinal infiltration, and poor presenting visual acuity and when there is no clinical improvement with systemic and intravitreal therapy. However, the role and timing of vitrectomy remain unclear in patients with endogenous endophthalmitis. Sheu et al. reported no significant relationship between vitrectomy and visual outcome in Klebsiella endophthalmitis. However, they suggested early vitrectomy should be considered in patients whose anterior chamber inflammation did not respond well to intravitreal antibiotics [28]. On the other hand, Yoon et al. demonstrated that following early vitrectomy for Klebsiella endogenous endophthalmitis, $50 \%$ achieved a vision of CF or better after 6 months [14]. Early vitrectomy performed within 10 days of the appearance of ocular symptoms or signs resulted in a better visual prognosis (CF or better) than without vitrectomy [17]. In other studies, early vitrectomy within 2 weeks of presentation in severe cases or suspected virulent organisms was associated with good overall outcome $[14,17]$. In our case series, 73 eyes $(51.4 \%)$ underwent vitrectomy. Vitrectomy was performed within 2 weeks in 38 eyes (52.1\%) and more than 2 weeks in 35 eyes (47.9\%). The most common indication for early vitrectomy was poor presenting visual acuity of $\mathrm{CF}$ or worse in 31 cases (81.6\%). Persistent or increased vitreous opacities or anterior chamber cells despite systemic and intravitreal antibiotics were other indications for early vitrectomy. There was no significant difference between early vitrectomy (within 2 weeks) compared to delayed vitrectomy (more than 2 weeks) for favorable visual prognosis $(p=0.327)$.

Generally, the visual outcome of endogenous endophthalmitis is poor due to early and extensive retinal involvement. Virulent causative organisms, poor host defense, misdiagnosis leading to delayed treatment, inadequate treatment, inappropriate therapy, and occurrence of complications such as panophthalmitis are associated with poor prognosis. Wu et al. reported that the eyes with bacterial endogenous endophthalmitis had a worse outcome compared to patients with fungal endophthalmitis [11]. Lim et al. concluded that gram-negative bacteria had worse visual outcomes compared to grampositive bacteria or fungus [15].

Visual outcomes in Klebsiella endophthalmitis has been poor despite treatment with a combination of systemic and intravitreal antibiotics [12, 13]. Case series and literature reviews involving infection with $\mathrm{K}$. pneumonia showed that visual acuity achieved was CF or better in $34.0 \%$ of eyes, and $16.0 \%$ had evisceration or enucleation [5]. Sheu et al. reported 19 eyes (35.8\%) had 
final visual acuity of CF or better [28]. Connell et al. found that all the patients in their study needing enucleation were infected by Klebsiella [1]. In our series, 100 eyes (73.0\%) achieved final visual acuity of CF or better. However, in cases with Klebsiella endogenous endophthalmitis, only 18 eyes (25.4\%) achieved final visual acuity of CF or better, which is comparable with other studies. Ten eyes were complicated with panophthalmitis, and 5 of them were due to Klebsiella pneumonia.

In our series, a good presenting visual acuity was the only prognostic factor associated with good visual outcomes of CF or better. Lim et al., Nishida et al., and Binder et al. in their case series also described that a good presenting visual acuity was significantly associated with good final visual acuity $[15,16,20]$. We found that DM, presence of a source of infection, organism, and intravitreal antibiotics were not related to poor visual outcome.

\section{Study limitation}

This study is limited by the retrospective design. As the data was collected retrospectively, some of the information was not available. Apart from that, patients with culture-negative result were also included in this study which may have included those with non-infectious uveitis. In the future, we may need to use other methods such as polymerase chain reaction (PCR) with higher sensitivity and specificity. Lack of uniform guidelines and treatment protocol is another limitation. Observational and prospective case series are needed in the future to assess long-term outcomes.

\section{Conclusions}

The visual prognosis of endogenous endophthalmitis (EE) is poor. Gram-negative organisms specifically Klebsiella pneumonia were the most common organisms isolated. Urinary tract infection was the main source of infection. Poor presenting visual acuity was significantly associated with poor visual outcomes.

\section{Abbreviations}

AGE: Acute gastroenteritis; CF: Counting fingers; EE: Endogenous Endophthalmitis; MRSA: Methicillin-resistant Staphylococcus aureus; PCR: Polymerase chain reaction

\section{Acknowledgements}

We would like to thank the Director General of Health Malaysia for his permission to publish this article. Last but not least, we express our gratitude to CRC Hospital Sultanah Nur Zahirah, Kuala Terengganu, and those who had extended their help in contributing to this manuscript.

\section{Authors' contributions}

All listed authors made a substantial contributions to the study. HI and AA participated in the design of the study. W and DS helped in the data collection. RM involved in the data collection, data analysis, literature search, and drafting, designing, and revising of the manuscript. SOM participated and supervised everything from the study concept and design until the manuscript revision. Each of the authors approved the submission of this version for publication. All authors read and approved the final version of the manuscript.
Ethics approval and consent to participate

Ethics approval was obtained by the Medical Research and Ethics Committee (MREC) prior to the initiation of the study.

\section{Consent for publication}

Not applicable.

\section{Competing interests}

The authors declare that they have no competing interests.

\section{Publisher's Note}

Springer Nature remains neutral with regard to jurisdictional claims in published maps and institutional affiliations.

\section{Author details}

${ }^{1}$ Department of Opthalmology, Hospital Sultanah Nur Zahirah, Kuala Terengganu, Terengganu, Malaysia. ${ }^{2}$ Department of Opthalmology, Hospital Sungai Buloh, Sungai Buloh, Malaysia. ${ }^{3}$ Department of Opthalmology, Hospital Selayang, Batu Caves, Malaysia. ${ }^{4}$ Department of Opthalmology, Hospital Kuala Lumpur, Kuala Lumpur, Malaysia. ${ }^{5}$ Department of Opthalmology, Hospital Shah Alam, Shah Alam, Malaysia.

Received: 5 July 2018 Accepted: 27 September 2018

Published online: 11 October 2018

\section{References}

1. Connell PP, O'Neill EC, Fabinyi D et al (2011) Endogenous endophthalmitis: 10-year experience at a tertiary referral centre. Eye 25(1):66-72. https://doi. org/10.1038/eye.2010.145

2. Okada AA, Johnson RP, Liles WC et al (1994) Endogenous bacterial endophthalmitis: report of a ten-year retrospective study. Ophthalmology 101:832-838

3. Chee SP, Jap A (2001) Endogenous endophthalmitis. Curr Opin Ophthalmol 12(6):464-470

4. Jackson TL, Eykyn SJ, Graham EM et al (2003) Endogenous bacterial endophthalmitis: a 17 year prospective series and review of 267 reported cases. Surv Ophthalmol 48(4):403-423

5. Wong JS, Chan TK, Lee HM, Chee SP (2000) Endogenous bacterial endophthalmitis: an East Asian experience and a reappraisal of a severe ocular affliction. Ophthalmology 107:1483-1491

6. Sharma S, Padhi TR, Basu S et al (2014) Endophthalmitis patients seen in a tertiary eye care centre in Odisha: a clinico-microbiological analysis. India J Med Res 139:91-98

7. Leibovitch I, Lai T, Raymond G et al (2005) Endogenous endophthalmitis: a 13 year review at a tertiary hospital in South Australia. Scand I Infect Dis 37: 184-189

8. Ratra D, Saurabh K, Das D et al (2015) Endogenous Endophthalmitis : a 10year retrospective study at a tertiary hospital in South India. Asia Pac J ophthalmol 4:286-292 doi:10.109/APO.0000000000000120

9. Zhang H, Liu Z (2010) Endogenous endophthalmitis: a 10-year review of culture positive cases in northern China. Ocul Immunol Inflamm 18:133-138. https://doi.org/10.3109/09273940903494717

10. Keswani T, Ahuja V, Changulani M (2006) Evaluation of outcomes of various treatment methods for endogenous endophthalmitis. Indian J Med Sci 60: $454-460$

11. Wu ZH, Chan RP, Luk FO et al (2012) Review of clinical features, microbiological spectrum and treatment outcomes of endogenous endophthalmitis over an 8-year period. J Ophthalmol 265078. https://doi. org/10.1155/2012/265078

12. Chen YJ, Kuo HK, Wu PC et al (2004) A 10-year comparison of endogenous endophthalmitis outcomes: an East Asian experience with Klebsiella pneumoniae infection. Retina 24:383-390

13. Yang CS, Tsai HY, Sung CS et al (2007) Endogenous Klebsiella endophthalmitis associated with pyogenic liver abscess. Ophthalmology $114: 876-880$

14. Yoon YH, Lee SU, Sohn JH, Lee SE (2003) Result of early vitrectomy for endogenous Klebsiella pneumoniae endophthalmitis. Retina 23:366-370

15. Lim HW, Shin JW, Cho HY et al (2014) Endogenous endophthalmitis in Korea, a six-year retrospective study. Retina 34:592-602. https://doi.org/10. 1097/AAE.0b013e3182a2e705 
16. Nishida T, Ishida K, Niwa Y et al (2015) An eleven-year retrospective study of endogenous endophthalmitis. J Ophthalmol. https://doi.org/10.1155/2015/ 261310

17. Lee S, Um T, Joe SG et al (2012) Changes in the clinical features and prognostic factors of endogenous endophthalmitis : fifteen years of clinical experience in Korea. Retina 32:977-984. https://doi.org/10.1097/ IAEOb013e318228e312

18. Mohammad AS, Muhammad H, Aniruddha A et al (2015) Endogenous endophthalmitis: diagnosis management and prognosis - review. Journal of Ophthalmic Inflammation and Infection 5:32. https://doi.org/10.1186/ s12348-015-0063-y

19. Schiedler V, Scott IU, Flynn HW Jr et al (2004) Culture-proven endogenous endophthalmitis: clinical features and visual acuity outcomes. Am J Ophthalmol 137:725-773. https://doi.org/10.1016/j.ajo.2003.11.013

20. Binder Ml, Chua J, Kaiser PK et al (2003) Endogenous endophthalmitis. An 18-years review of culture-positive cases at a tertiary care center. Medicine 82:97-105

21. Ness T, Pelz K, Hansen LL (2007) Endogenous endophthalmitis: microorganism, disposition and prognosis. Acta Ophthalmol 85:852-856

22. Lingappan A, Wykoff CC, Albini TA, et al (2012) Endogenous fungal endophthalmitis: causative organisms, management strategies, and visual acuity outcomes. Am J Ophthalmol 153(1):162-166. doi:https://doi.org/10. 1016/j.ajo.2011.06.020, e161

23. Sridhar J, Flynn HW Jr, Kuriyan AE, et al (2013) Endogenous fungal endophthalmitis: risk factors, clinical features, and treatment outcomes in mold and yeast infections. J Ophthalmic Inflamm Infect 3(1):60.doi:https:// doi.org/10.1186/1869-5760-3-60

24. Nakayama F, Soloway RO, Nakama T et al (1986) Hepatolithiasis in East Asia. Retrospective study. Dig Dis Sci 31:21-26

25. Kowalski RP, Karenchak LM, Eller AW (1993) The role of ciprofoxacin in endophthalmitis therapy. Am J Ophthalmol 116:695-699

26. Lesar TS, Fiscella RG (1985) Antimicrobial drug delivery to the eye. Drug Intell Clin Pharm 19:642-654

27. Yonekawa Y, Chan RV, Reddy AK et al (2011) Early intravitreal treatment of endogenous bacterial endophthalmitis. Clin Exp Ophthalmol 39(8):771-778. https://doi.org/10.1111/j.1442-9071.2011.02545.x

28. Sheu SJ, Kung YH, Wu TT et al (2011) Risk factors for endogenous endophthalmitis secondary to Klebsiella pneumonia liver abscess: 20 year experience in southern Taiwan. Retina 31:2026-2031. https://doi.org/10. 1097/IAE.0b013e31820d3f9e

\section{Submit your manuscript to a SpringerOpen ${ }^{\circ}$ journal and benefit from:}

- Convenient online submission

- Rigorous peer review

- Open access: articles freely available online

- High visibility within the field

- Retaining the copyright to your article

Submit your next manuscript at $\boldsymbol{\nabla}$ springeropen.com 